\title{
Gestalt grouping effects on tactile information processing: when touching hands override spatial proximity
}

\author{
Christian Frings $\cdot$ Charles Spence
}

Published online: 10 January 2013

(C) Psychonomic Society, Inc. 2013

\begin{abstract}
Using a tactile variant of the negative-priming paradigm, we analyzed the influence of Gestalt grouping on the ability of participants to ignore distracting tactile information. The distance between participants' hands, to which the target and distractor stimuli were simultaneously delivered, was varied (near/touching hands vs. hands far apart). In addition, the influence of touching hands was controlled, as participants wore gloves and their hands were blocked from vision by a cover. The magnitude of the tactile negative-priming effect was modulated by the interaction between hand separation and whether or not gloves were worn. When the hands were touching, negative priming emerged only while wearing gloves that prevented direct skin-to-skin contact. In contrast, when the separation between the participants' hands was larger, negative priming emerged only when gloves were not worn. This pattern of results is interpreted in terms of the competing influences of two interacting Gestalt principles-namely, connectedness and proximity - on the processing of tactile distractors.
\end{abstract}

Keywords Touch · Grouping and segmentation ·

Negative priming

At every waking moment, a wealth of information from the various sensory systems is processed by our brains. However, the efficient control of action requires that only certain features of particular stimulus representations be fully processed, and subsequently used to guide our actions (e.g.,

C. Frings $(\bowtie)$

Department of Psychology, Cognitive Psychology,

University of Trier, Campus I,

54286 Trier, Germany

e-mail: chfrings@uni-trier.de

\section{Spence}

Crossmodal Research Laboratory, Department of Experimental

Psychology, University of Oxford, South Parks Road,

Oxford OX1 3UD, UK
Allport, 1987). It is now widely believed that selective attention helps us to find, and preferentially process, relevant information at the expense of less relevant information. In fact, selection is considered to represent one of the principal functions of attention (see Pashler, 1998; Spence, 2010). By and large, it has been assumed that selection is achieved through the facilitation of the processing of relevant stimuli and/or the inhibition (or ignoring) of irrelevant stimuli. In the present article, the focus is on the latter process - that is, on the ignoring of irrelevant tactile information (i.e., distractors).

Abundant evidence now supports unimodal selection in the visual and auditory modalities (see, e.g., Allport, 1993; Johnston \& Dark, 1986; Spence, 2010). Yet, to date, few studies have attempted to analyze distractor processing within the tactile modality (see Frings, Amendt, \& Spence, 2011; Frings, Bader, \& Spence, 2008; Frings \& Spence, 2010; Gallace \& Spence, in press; Moeller \& Frings, 2011; Spence \& Gallace, 2007). So far, it seems that established experimental paradigms such as the negative-priming task (Tipper, 1985; see Fox, 1995, for a review), which are assumed to tap the ignoring of distractors, work just as effectively in touch as in vision. Intriguingly, though, the magnitude of negative priming seems to be larger in the tactile modality than in vision (cf. Frings et al., 2011).

In a similar vein, stimulus-response binding effects have been demonstrated in the tactile modality that are comparable to those documented previously in vision (see Zmigrod, Spapé, \& Hommel, 2009). In fact, the integration of stimulus features (irrespective of feature modality) with response features into coherent stimulus-response episodes appears to be modality-independent. For example, repeating a visual stimulus with a certain response will lead to beneficial effects if the response is also repeated; the same holds true for tactile stimuli (Zmigrod et al., 2009). Furthermore, Moeller and Frings (2011) recently provided evidence demonstrating the influence of stimulus-response binding on the processing of tactile distractors; that is, the co-occurrence of 
a tactile distractor with a particular response leads to the integration of the distractor and the response into one memory entry that could be retrieved by another presentation of the distractor. Overall, distractor processing in the tactile modality appears to be comparable (albeit by no means identical) to the distractor processing that has been documented previously in the visual modality. That said, further research will certainly be needed in order to understand the processing of tactile distractors in anything like as much detail as we already have for vision. In the study reported here, we analyzed how specific perceptual mechanismswhich we interpret in terms of Gestalt grouping mechanisms (see Gallace \& Spence, 2011) - might affect the selective processing of tactile distractors.

The Gestalt grouping principles were originally formulated more than a century ago by researchers working in the field of visual perception (e.g., von Ehrenfels, 1890; Wertheimer, 1912). Extensive research by Gestalt psychologists on the topic of unimodal perceptual organization has revealed the existence of a number of different grouping principles - such as spatial proximity, similarity, good continuation, common fate (or uniform destiny), closure, and Einstellung (or "set")that appear to exert a profound influence on people's perception of the organization of visual displays (e.g., Koffka, 1935; Köhler, 1930; Smith, 1988; Wagemans et al., 2012; Wertheimer, 1923/1938). Following on from the original studies in the visual modality, researchers have subsequently been able to demonstrate that many, if not all, of the Gestalt grouping principles also apply to the auditory modality as well (for reviews, see Bregman, 1990; Dyson, 2009; Julesz, 1971; Wagemans, 2012), not to mention to the tactile modality (see Gallace \& Spence, 2011, for a recent review).

Generally speaking, it is assumed that Gestalt grouping principles further the selection of relevant as opposed to irrelevant information, as they help to separate targets from distractors on the basis of varying properties such as proximity, similarity, closure, and common fate (see, e.g., Driver \& Baylis, 1989; Egly, Driver, \& Rafal, 1994; Kahneman \& Henik, 1981; Lamers \& Roelofs, 2007; Reynolds, Kwan, \& Smilek, 2010). For example, Driver and Baylis (see also Reynolds et al., 2010) have demonstrated that more distant distractors that just so happen to move in the same direction as a target stimulus elicit stronger compatibility effects than do nearer distractors not moving in that direction-thus overriding the immediate spatial proximity between the target and the distractor (i.e., the principle of common fate presided over the principle of proximity, and over any influence of spatial attention under the particular stimulus conditions used in this study). In a similar vein, some evidence has indicated that Gestalt grouping principles affect the deployment of selective attention in the Stroop task (see, e.g., Lamers \& Roelofs, 2007; see also Davis \& Driver, 1994). In addition, Frings and Rothermund (2011) recently demonstrated that the stimulus-response bindings of responses to targets and to distractor stimuli accompanying these targets are influenced by the principles of Gestalt grouping. Specifically, binding effects for distractors were larger if the distractors were perceived as belonging to the target as a result of perceptual grouping.

In the present study, we were interested in the influence of Gestalt grouping principles on the processing of tactile distractors. In particular, we analyzed whether the distance in external space between targets and distractors and the touching of the hands to which the stimuli were delivered would influence the processing of tactile distractors. We interpreted these experimental manipulations in terms of the Gestalt principles of spatial proximity and connectedness. There is evidence that both principles can work in the tactile modality. In particular, the Gestalt principle of spatial proximity (i.e., elements that are close together group together) have been observed in touch when the eccentricities from which different tactile stimuli were presented have been manipulated (e.g., Chang, Nesbitt, \& Wilkins, 2007). In addition, the apparent-motion illusion can be elicited in the tactile modality on the basis of the proximity of the stimulated elements (e.g., Harrar \& Harris, 2007; Lakatos \& Shepard, 1997; see also Carter, Konkle, Wang, Hayward, $\&$ Moore, 2008). The Gestalt principle of connectedness (e.g., elements that are connected group together-i.e., as a unit) has also been observed in touch. In fact, research reported by Haggard, Kitadono, Press, and Taylor-Clarke (2006) demonstrated a significant influence of touching versus nontouching hands on the detection and identification of tactile stimuli delivered to the hands and/or digits. In terms of the principles of Gestalt grouping, one may interpret this as some kind of grouping based on connectedness (see Gallace \& Spence, 2011, for a review).

In the present study, we adopted the tactile negativepriming paradigm from Frings et al. (2008). In each trial, the participants had to try to identify the vibrotactile target stimulus while simultaneously trying to ignore the distractor vibration. Slower responses were expected in those trials in which a vibrotactile stimulus that had been ignored in the preceding trial (the prime) constituted the target in the current trial (the probe). ${ }^{1}$ In addition, the separation between the target and distractor vibrations in external space was varied. When the distance between the target and distractor is large, participants normally find it easier to select the target (and to ignore the distractor), according to the

\footnotetext{
${ }^{1}$ Negative priming is assumed to involve the ignoring of prime distractors. That said, the underlying mechanism(s), be they inhibition (e.g., Houghton \& Tipper, 1994) or the retrieval of a "do-not-respond" tag (e.g., Neill, 1997), are still being debated (e.g., Tipper, 2001). For the purposes of understanding the influence of the Gestalt grouping principles on distractor processing in the tactile modality, we can, however, ignore this debate.
} 
principle of proximity, as compared to when the distance between the target and distractor is small (see Kahneman \& Treisman, 1984). Tactile negative priming should, in turn, be more pronounced in the condition in which the distance between the target and distractor is small, as compared to a condition with a large distance in external space, as it is known that the negative-priming effect increases with increasing selection difficulty (Frings \& Spence, 2011; Frings \& Wühr, 2007; Houghton \& Tipper, 1996). Evidence from experiments conducted in the visual modality also provides support for this argument. In these studies, the Gestalt grouping principles influenced visual negative priming, in that distractors that grouped together with the target elicited larger negativepriming effects than did distractors that were not grouped with the target (Fox, 1998; Yeh \& Chao, 2004; but see Fuentes, Humphreys, Agis, Carmona, \& Catena, 1998).

However, in the condition in which the distance between the hands was small, the hands did actually touch each otherthat is, the index fingers as well as the thumbs of the left and right hands touched, thus possibly leading to the impression that the vibrations originated from the same object. In terms of the principles of Gestalt grouping, one may therefore interpret this as some kind of grouping by connectedness (see Gallace \& Spence, 2011, for a review). By comparing results from the near conditions with or without touching hands (or the impression thereof), we could judge the possible influence of connectedness on the processing of distractors and analyze the influence of proximity without the confound of touching versus nontouching hands.

\section{Experiment 1}

In this experiment, four different vibrotactile patterns were presented in the context of a target identification task. One target and one distractor vibrotactile stimulus were presented simultaneously in each trial until participants responded to the target stimulus. The location of the target was unpredictable and was cued by the color of the display at the start of each trial. Importantly, each vibrotactile pattern was mapped to a specific finger (not to a response key) for responding. After the first block of trials in the experiment, the participants' hands were placed in another location (such that the spatial distance between the hands could be manipulated). A learning phase was presented prior to the experimental phase in order to reduce participants' error rates. Note that the procedure and apparatus/materials closely followed those described in Frings et al. (2008).

\section{Methods}

Participants A group of 14 students from Saarland University took part in this experiment for a payment of $€ 8$. Their median age was 23 years (ranging from 19 to 33 years). All of the participants had a normal sense of touch and normal or corrected-to-normal vision by self-report. Also, all of the participants were naïve as to the purpose of the experiment.

Design Essentially, the experiment consisted of a 2 (priming condition: ignored repetition [IR] vs. control [C] $) \times 2(\mathrm{ex}-$ ternal distance between target and distractor: near vs. far) within-participants design. The sequence of external distances between targets and distractors (near $\rightarrow$ far vs. far $\rightarrow$ near) was varied between participants. Negative-priming effects were computed as the difference in performance between control and ignored-repetition trials.

Apparatus and materials The experiment was conducted on a standard PC with a 17-in. CRT screen. Vibrotactile stimuli were delivered by means of two skin transducers (Model VBW32, Audiological Engineering Corporation). Each transducer weighed $6.5 \mathrm{~g}$ (including the Velcro strip used to fasten it to the participant's hand) and was $2.54 \mathrm{~cm} \times$ $1.85 \mathrm{~cm} \times 1.06 \mathrm{~cm}$ in size. The transient response of each transducer was measured at $5 \mathrm{~ms}$ (attack and decay). The amplitude range extended to $50 \mathrm{~dB}$ above the average sensory threshold. E-Prime (Version 1.1) was used to conduct the experiment. The software was used to control the presentation of the sound files. A Sony hi-fi system was used to amplify the sounds. Instead of normal loudspeakers, however, the skin transducers were connected to the amplifier. Thus, the sound files were used to generate vibrotactile stimuli presented via the transducers. Four tones with different rhythms were used to produce the vibrotactile stimuli. The signals were created using the freeware program $\mathrm{Au}$ dacity (Version 1.2.6). The stimuli were presented repeatedly until the participant responded or until $5,000 \mathrm{~ms}$ had elapsed since the start of the trial. One stimulus consisted of a single longer pulse $(340 \mathrm{~ms})$ followed by three shorter pulses (180 ms each). There were two pauses of $60 \mathrm{~ms}$ each, one after the longer pulse and one after the last of the three short pulses. The duration of the whole sequence was $1,000 \mathrm{~ms}$. Another of the stimuli comprised four pulses of equal length (192 ms each) and intensity, with each pulse being followed by a $58-\mathrm{ms}$ empty interval. The third stimulus was composed of one short pulse $(110 \mathrm{~ms})$ followed by an 890-ms pause. The fourth stimulus simply consisted of a continuous vibration presented until participants reacted or 5,000 ms had elapsed. ${ }^{2}$

\footnotetext{
${ }^{2}$ The four stimuli differed in terms of at which point in time they could be safely identified, as well as in processing difficulty. However, as probe target type was orthogonally varied with respect to all other factors, these issues were not problematic. In addition, negative priming was not significantly different with respect to stimulus type.
} 
Procedure The participants were tested individually in a quiet and dimly lit room. Each participant sat $60 \mathrm{~cm}$ in front of the PC, and the skin transducers were fixed onto his or her palms. The transducers were placed on the side of each of the participant's palms. Participants had to identify the target vibrations by responding with the appropriate finger. The four response fingers were placed by the experimenter on a QWERTY keyboard in front of the participant. To limit the noise made by the transducers touching the table, we used armrests on which the participant put his or her wrists. Instructions were given on the screen and summarized by the experimenter at the beginning of each phase of the experiment. White noise was presented over headphones to mask the sounds made by the operation of the vibrotactile stimulators (see Fig. 1).

The experimental session comprised four training phases, in which the assignments of the vibrotactile stimuli to specific finger responses were learned, and an experimental phase, in which the participants had to perform the negative-priming task itself.

In the experimental phase, each trial consisted of the following events: The participants initiated each trial by pressing the space bar. Next, the prime was presented. This comprised two stimuli (i.e., the target vibration and the distractor vibration). Both stimuli were simultaneously presented until the participant responded. The participants were instructed to identify the target and to ignore the vibrotactile distractor stimulus. They were also told to respond as rapidly and accurately as possible. Simultaneous with the onset of the stimuli, the display changed color; the screen also remained colored until the participants reacted. A red screen indicated that the target stimulus would be presented to the participant's right hand, whereas a blue screen indicated that the target would be presented to the left hand. The Velcro strips (with which the tactile stimulators were taped to the palm) were colored in a congruent manner (i.e., the Velcro strip on the participant's right hand was red, whereas the Velcro strip of the left hand was blue). Thereafter, a white screen was displayed for $1,000 \mathrm{~ms}$, followed by the probe. The probe also consisted of two stimuli, one the target and the other the distractor. Again, the participants had to identify the target vibration that was indicated by the color of the screen.

Importantly, in the condition with targets and distractors separated by a small distance in external space, the two centers of the palm surfaces were approximately $10 \mathrm{~cm}$ apart. In this condition, the experimenter placed the participant's left index finger on the "B" key, the left middle finger on the "V" key, the right index finger on the " $\mathrm{N}$ " $\mathrm{key}$, and the right middle finger on the " $\mathrm{M}$ " key. In the condition in which the targets and distractors were separated by a large distance in external space, the two centers of the palm surfaces were approximately $40 \mathrm{~cm}$ apart. In this condition, the experimenter placed the participant's left index finger on the " $X$ " key, the left middle finger on the " $Z$ " key, the right index finger on the " 2 " key of the number pad, and the right middle finger on the " 3 " key of the number pad.

All possible combinations of each probe with two different stimuli were constructed while orthogonally varying the positions of the stimuli, resulting in 24 different probes. Since we were only interested in the ignored-repetition (IR) and control (C) conditions, we built all possible primes for each probe that would transfer this prime-probe sequence into an IR or C trial (four IR primes and four $\mathrm{C}$ primes for each probe). In an IR trial, the prime distractor
Fig. 1 Procedural details of the test arrangement (see the main text for details). The distance between the participant's hands was measured as the distance between the centers of the left and right hands (depicted as black circles). In the near distance condition, the participant's hands were touching in Experiments 1 and 2
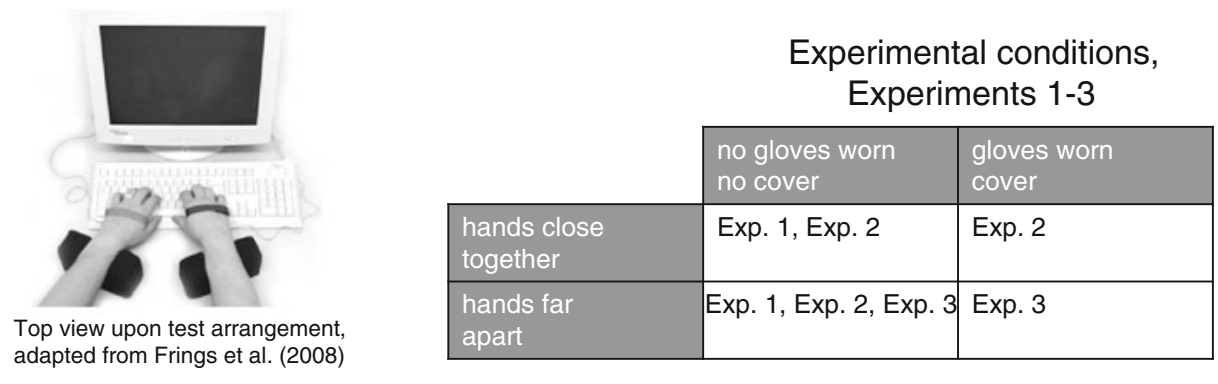

Gloves and cover

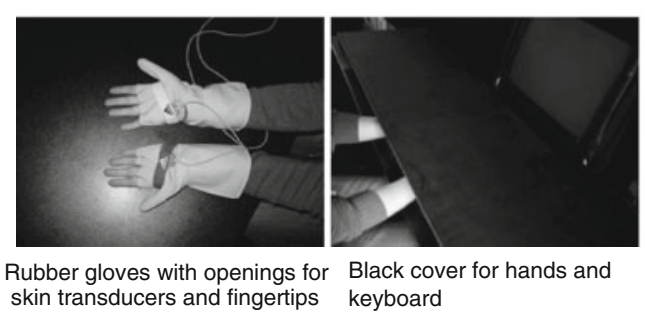

Hand separation
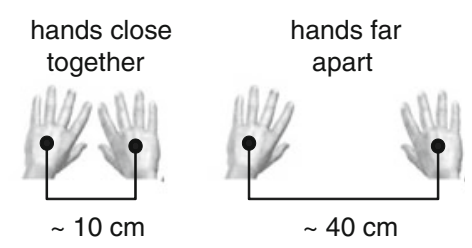
was repeated as the probe target, whereas in a $\mathrm{C}$ trial, all four stimuli were different. Note that the positions of the targets as well as the response hands were orthogonally varied with respect to all other factors (cf. Frings et al., 2008). This design resulted in 192 experimental trials (96 IR and $96 \mathrm{C}$ trials). The trials were presented in a pseudorandomized fashion for each distance condition.

Half of the participants started the experiment with their hands arranged in the far distance condition, whereas the other half of the participants started with their hands in the near distance condition. After the first 96 experimental trials, the participants could rest for a short period (1$3 \mathrm{~min}$ ). Thereafter, the experimenter placed the participant's response fingers according to the other distance condition; that is, if the participant had started with the near condition, her or his fingers were placed according to the far distance condition arrangement in the second block of the experiment. Participants received 12 training trials before each experimental block, in addition to the stimulus-response mapping learning phases (see below).

A learning phase was conducted at the start of the experiment, in which the participants learned the assignments of the vibrotactile stimuli to the response fingers. In each trial of Learning Phase 1, one of the vibrations was randomly presented to either hand. Simultaneously, an image was displayed on the screen that showed a photograph of one left and one right hand. One finger was colored green, indicating the corresponding response finger for this vibration. The background of this image was white. The trial ended when the participant responded with the particular finger. Each vibration was presented three times to each hand (i.e., 24 trials in total). We used photographs for a direct mapping of responses to vibrotactile patterns, thereby minimizing possible strategies (e.g., verbal recoding of the tactile patterns) on the part of the participants. It was also stressed that the participant's fingers would be placed on different keys during the course of the experiment, and hence that the participant would have to learn the stimulus-finger association. In Learning Phase 2, the participants were given practice in attending to a specific target hand. The procedure was the same, except that the background of the display was now either red or blue. When red, the participant sensed the vibration on his or her right hand, and when it was blue, he or she sensed it on the left hand. This phase of the experiment also consisted of 24 trials. In Learning Phase 3 , we tested whether the participants had learned the correct stimulus-response mappings between the vibrotactile stimuli and the fingers that they had to respond with. Once again, the color of the screen (red or blue) indicated on which hand the target vibration would be presented. The participants were instructed to try to identify the vibration and respond with the appropriate finger. This time, however, the photograph of the two hands indicating the correct finger was no longer displayed. Feedback concerning the correctness of a participant's responses was provided after each trial. The four vibrations were presented repeatedly in a random order until the participants were able to correctly identify each of the vibrations five times in a row (on average, participants needed $12 \mathrm{~min}$ to fulfill this learning criterion). Finally, in Learning Phase 4, the participants were presented with 48 trials containing both targets and distractors. These trials were exactly the same as the experimental trials, except that participants were still given feedback about the correctness of their answers. The length of all four learning phases was about $20-25 \mathrm{~min}$ in total.

\section{Results}

Only correct probe reaction times (RTs) were analyzed (the error rate on the probe displays was $25.5 \%$ ). Moreover, RTs had to be longer than $350 \mathrm{~ms}$ and lower than the Tukey outlier criterion (1.5 interquartile ranges above the third quartile, Tukey, 1977; this criterion was 4,036 ms, leading to $1.9 \%$ of the trials being discarded as outliers). With respect to these criteria, $27.0 \%$ of the trials were discarded. The mean RTs and error rates are highlighted in Table 1.

Reaction times The sequence of the external distance condition showed no main effect or interaction effect except the interaction of sequence with external distance (meaning that participants were always somewhat slower in their first than in their second block). Thus, for the sake of simplicity we dropped the factor sequence of distance conditions. Correct probe RTs were subjected to a 2 (priming condition: IR vs. C) $\times 2$ (external distance: near vs. far) multivariate analysis of variance (MANOVA). The main effect of priming condition was not significant, $F(1,13)=1.98, p=.183, \eta_{\mathrm{p}}{ }^{2}=$ .13 , indicating no average negative-priming effect. In addition, the main effect of external distance was not significant, $F(1,13)=0.0, p=.787, \eta_{\mathrm{p}}{ }^{2}=.006$, showing that participants' RTs were not different in these conditions. Importantly, the interaction between external distance and priming condition was marginally significant, $F(1,13)=3.00, p=.053$ (one-

Table 1 Mean reaction times (in milliseconds) and percentages of errors (in parentheses) as a function of priming condition and external distance in Experiment 1

\begin{tabular}{lll}
\hline & \multicolumn{2}{l}{ External Distance } \\
\cline { 2 - 3 } Priming Condition & Near & Far \\
\hline Ignored repetition (IR) & $1,971[299]$ & $2,034[363]$ \\
& $(22.9)[18.5]$ & $(21.9)[17.2]$ \\
Control (C) & $2,001[347]$ & $1,956[301]$ \\
& $(20.2)[16.1]$ & $(16.9)[13.7]$ \\
\hline
\end{tabular}

Standard deviations appear in square brackets 
tailed), $\eta_{\mathrm{p}}{ }^{2}=.19$, thus showing that negative-priming effects differed as a function of external distance. In particular, in the near distance condition, a trend toward a positive- instead of a negative-priming effect was observed, $M=25 \mathrm{~ms}$ ( $S D=$ $175 \mathrm{~ms}$ ), that was not significantly different from zero, $t(13)=0.53, p=.606$; however, in the far distance condition, a significant negative-priming effect of $M=-93 \mathrm{~ms}(S D=$ $134 \mathrm{~ms}$ ) was observed, $t(13)=2.60, p=.022$ (cf. Fig. 2).

Error rates We conducted the same MANOVA on the error data. However, neither the main effects nor their interaction was significant, all $p \mathrm{~s}>.15$.

\section{Discussion}

The results of Experiment 1 demonstrate a modulation of tactile negative priming due to the variations in the distance between the target and distractor vibrations in external space while the internal (or somatotopic) distance was held constant: In a condition with a larger distance between the hands, we found negative-priming effects comparable to the effect observed in Frings et al. (2008) using a middle distance. Yet, when the distance between the hands was small, tactile negative priming diminished to a nonsignificant level. This result is at odds with experiments in vision, which usually show that a smaller distance between targets and distractors enhances the interference from the distractor and, in turn, requires stronger inhibition/ignoring of the distractor. As a result, one would have expected larger negative priming with a smaller external distance between targets and distractors.

Yet, in the near condition, the hands actually did touch. Thus, it seemed possible that the connectedness of the hands may have overridden the effect of spatial proximity. Before

\section{Negative Priming Effects (in ms), Experiments 1-3}

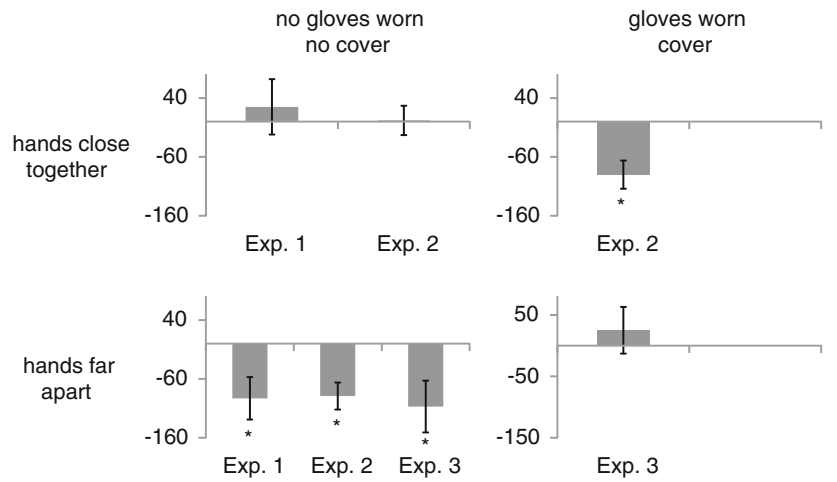

Fig. 2 Negative-priming effects (in milliseconds) as a function of distance between the hands and wearing gloves/using cover, Experiments $1-3$. The error bars depict the standard errors of the means. "Significant negative-priming effects $(p<.05)$ elaborating on this effect, we tested this assumption in a second experiment, in which we replicated the near condition while participants wore gloves, thereby preventing the impression of touching hands.

\section{Experiment 2}

In Experiment 2, we conducted the near condition from Experiment 1 with or without touching hands (or the impression thereof), and thus we could judge the possible influence of connectedness on the processing of tactile distractors and analyze the influence of proximity without having to worry about the potentially confounding influence of whether or not the participants' hands were actually touching.

\section{Method}

Participants A group of 38 students from Saarland University or the University of Trier took part for course credit. Their median age was 22 years (ranging from 20 to 49 years). All of the participants had normal touch and normal or corrected-to-normal vision by self-report. The data from three of the participants were discarded (two had high error rates that were outliers according to Tukey, 1977, when compared with the error rate of the full sample, and the data from one additional participant had to be excluded due to technical issues while delivering the tactile patternsi.e., the amplifier was disconnected, and in turn, the vibrations were hard to identify).

Design Essentially, the experiment consisted of a 2 (priming condition: IR vs. C) $\times 3$ (external distance between target and distractor: far vs. near vs. near with gloves) withinparticipants design. The sequence of external distance between targets and distractors was counterbalanced across participants. In addition, prime target position and probe target position were also varied orthogonally with respect to the other factors but were collapsed in the analysis. Negative-priming effects were computed as the difference in performance between C and IR trials.

Apparatus, materials, and procedure Experiment 2 was a replication of Experiment 1, with the following exceptions. E-Prime (Versions 1.1 and 2.0) and a Sony STR-DN1030 or a Tangent AMP-50 hi-fi system were used to conduct the experiment. We conducted three conditions: far without gloves, near without gloves (both as in Exp. 1), and near with gloves. In the near condition with gloves, participants wore rubber gloves with openings for the skin transducers to contact a participant's palms directly. In addition, a black cover sheet prevented participants from viewing their hands 
(cf. Fig. 1). This design resulted in 288 experimental trials (144 IR and 144 C trials). In an IR trial, the prime distractor was repeated as the probe target, whereas in a $\mathrm{C}$ trial, all four stimuli were different. The trials were presented in a pseudorandomized order for each condition. The three conditions (near with and without gloves, and far) were counterbalanced between participants.

\section{Results}

Only correct probe RTs were analyzed (the error rate on probe displays was $15.6 \%$ ). Moreover, RTs had to be longer than $350 \mathrm{~ms}$ and below the Tukey outlier criterion (1.5 interquartile ranges above the third quartile, Tukey, 1977; in the present experiment, this criterion was $3,664 \mathrm{~ms}$, and $4.1 \%$ of trials were discarded due to the outlier criterion). Excluding outliers and error probes resulted in the elimination of $17.8 \%$ of the trials. The mean RTs and error rates are shown in Table 2. The sequence of the distance conditions showed no main effect or interaction, except the interaction of sequence with external distance (which meant that participants were always somewhat slower in their first than in their second or third block). Thus, for the sake of simplicity we dropped this factor (i.e., sequence of distance conditions) from further analysis.

Reaction times Correct probe RTs were subjected to a 2 (priming condition: IR vs. C) $\times 3$ (external distance: far vs. near vs. near with gloves) MANOVA. The main effect of priming condition was significant, $F(1,34)=15.06, p<$ $.001, \eta_{\mathrm{p}}{ }^{2}=.31$, indicating an average negative-priming effect. Yet the main effect of external distance was not significant, $F(2,33)=2.29, p=.127, \eta_{\mathrm{p}}{ }^{2}=.012$, showing that participants' RTs were not different in these conditions. The interaction between external distance and priming condition was significant, $F(2,33)=3.51, p=.041, \eta_{\mathrm{p}}{ }^{2}=.18$, thus showing that the negative-priming effects differed as a function of the external distance between the participant's hands. In addition to the nonspecific interaction effect, we built contrasts according to our hypothesis concerning the

Table 2 Mean reaction times (in milliseconds) and percentages of errors (in parentheses) as a function of priming condition and external distance in Experiment 2

\begin{tabular}{llll}
\hline & \multicolumn{2}{l}{ External Distance } & \\
\cline { 2 - 4 } Priming Condition & Near & Far & Near + Gloves \\
\hline Ignored & $1,897[398]$ & $1,982[400]$ & $2,014[400]$ \\
repetition (IR) & $(16.4)[11.4]$ & $(16.0)[9.8]$ & $(17.1)[12.6]$ \\
Control (C) & $1,899[397]$ & $1,888[366]$ & $1,923[401]$ \\
& $(15.5)[10.4]$ & $(12.8)[8.0]$ & $(16.2)[11.8]$ \\
\hline
\end{tabular}

Standard deviations appear in square brackets interaction of distance and priming condition. In fact, the negative-priming effect in the near distance condition was significantly different from the negative-priming effects observed in either the far distance condition, $F(1,34)=5.27$, $p=.028, \eta_{\mathrm{p}}^{2}=.13$, or the near-with-gloves condition, $F(1,34)=6.39, p=.016, \eta_{\mathrm{p}}^{2}=.16$, whereas the nearwith-gloves condition and the far condition did not differ, $F<1$. This pattern of results was also reflected when the simple negative-priming effects in the three distance conditions were tested against zero (cf. Fig. 2). In particular, in the near distance condition, no significant negative-priming effect was observed, $M=2 \mathrm{~ms}(S D=145 \mathrm{~ms}), t(34)=0.06$, $p=.953$. In contrast, in the far and gloves conditions, significant negative-priming effects were observed: $M=-90 \mathrm{~ms}(S D=144 \mathrm{~ms}), t(34)=3.71, p=.001$, for the gloves condition, and $M=-89 \mathrm{~ms}(S D=181 \mathrm{~ms}), t(34)=$ $3.06, p=.004$, for the far condition.

Error rates We conducted the same MANOVA on the error data. However, only the main effect of priming condition was significant, $F(1,34)=8.14, p<.007, \eta_{\mathrm{p}}{ }^{2}=.19$, indicating an average negative-priming effect in the error data, as well.

\section{Discussion}

The results of Experiment 2 replicated the main finding of Experiment 1: Tactile negative priming was modulated by variations in the distance between the target and distractor vibrations in external space. In a condition with a larger distance between the hands, we found negative-priming effects, while when the distance between the hands was small, tactile negative priming diminished to a nonsignificant level. Yet, intriguingly, if participants wore gloves and their hands were blocked from vision, negative-priming effects emerged even when the distance was small. In other words, the touching of the participant's hands overrode the principle of spatial proximity. This result appears to be in line with previous reports on the effects of touching hands (cf. Haggard et al., 2006). However, the effects of gloves and blocking the hands from vision could not safely be interpreted as long as their influence on tactile negative priming in the far condition was not analyzed. In addition, it seemed worthwhile to analyze participants' impressions of wearing gloves. We analyzed the effects of wearing gloves in the far condition in Experiment 3, and analyzed the impression of wearing gloves by getting a group of participants to complete a questionnaire.

\section{Questionnaire on the effects of wearing gloves}

With this questionnaire, we analyzed participants' impressions of wearing the gloves (and blocking their hands from 
vision). Our hope was that the subjective impressions of people would help to interpret the effects of gloves and a visual occluder on the tactile negative-priming effect.

\section{Method}

Participants A group of 31 students from the University of Trier took part for course credit. Their median age was 21 years (ranging from 19 to 49 years). All of the participants had normal touch and normal or corrected-to-normal vision by self-report.

Materials and procedure The participants were tested individually, seated in the laboratory in which Experiment 2 had taken place. The participants placed their hands on the response keys of the near condition as in Experiment 2 and answered six questions concerning their subjective impressions associated with the touching of their hands (see Fig. 3 for details). All questions were asked twice, once while the participants wore the same gloves as in Experiment 2 and had a black cover occluding their hands from view, and once without the gloves and cover. The order of conditions (with or without gloves and cover) was counterbalanced across participants.

\section{Results and discussion}

For each item, we computed the difference with respect to whether or not the participants wore gloves while answering the question. These six differences were submitted to a MANOVA, and thereby tested against the null vector. The constant term was significant, $F(6,25)=3.62, p=.010, \eta_{\mathrm{p}}{ }^{2}=$ .47 , demonstrating that the two conditions differed. Planned contrasts showed that Items 2 (If you move your right ring finger, how strongly do you feel this movement with your left hand?), 3 (How strongly do you feel that your hands touch the same object?), and 4 (How far are your hands apart?) differed significantly between the two conditions, with $F(1,30)=5.35$, $p=.028, \eta_{\mathrm{p}}{ }^{2}=.15 ; F(1,30)=3.42, p=.037$ (one-tailed), $\eta_{\mathrm{p}}{ }^{2}=$ .10 ; and $F(1,30)=6.13, p=.019, \eta_{\mathrm{p}}{ }^{2}=.17$, for Items 2,3 , and 4 , respectively. The other three items did not differ, $F_{\mathrm{s}}<1$.

Taken together, the results of the subjective rating questionnaire suggest that wearing gloves and blocking the participant's hands from view gave participants the impression that their hands seemed to be farther away and less connected.

\section{Experiment 3}

\section{Method}

Participants A group of 16 students from the University of Trier took part for course credit. Their median age was 22 years (ranging from 20 to 49 years). All of the participants had normal touch and normal or corrected-to-normal vision by self-report.

Design Essentially, the experiment consisted of a 2 (priming condition: IR vs. C) $\times 2$ (gloves/cover: with gloves and with cover vs. without gloves and without cover) withinparticipants experimental design. The sequence of blocks with or without gloves/cover was counterbalanced across participants. In addition, prime target position and probe target position were also varied orthogonally with respect to the other factors but collapsed in the analysis. Negativepriming effects were computed as the difference in performance between $\mathrm{C}$ and IR trials.
Fig. 3 Six items of the questionnaire on the proprioceptive impressions of both hands. The light gray columns depict means for the condition without gloves and cover, while the dark gray columns depict means for the condition with gloves and cover. The error bars depict the standard errors of the means

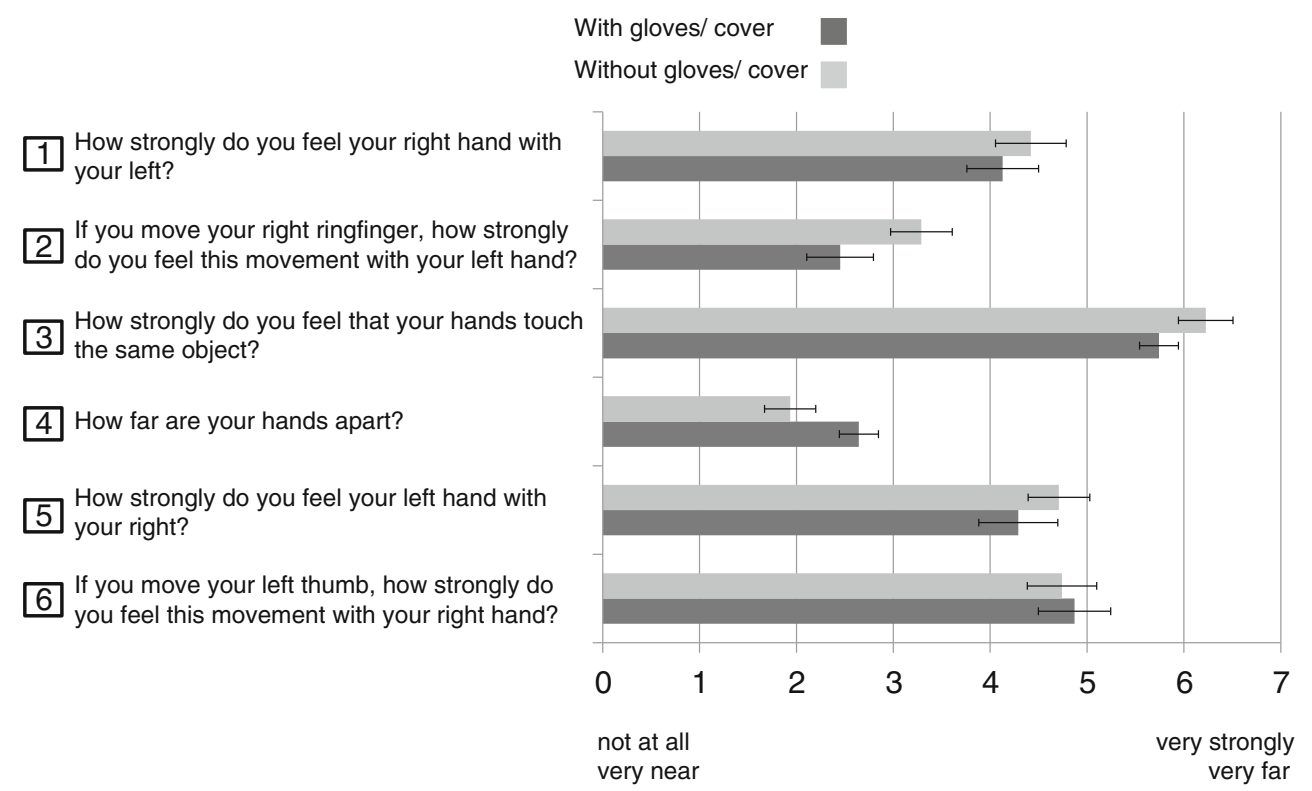


Apparatus, materials, and procedure Experiment 3 was an exact replication of the far condition of Experiment 1. However, in one block of trials, the participants wore the gloves and a cover was used to block their hands from view (exactly as in the near-with-gloves condition of Exp. 2), whereas in the other block, the participants worked through the far conditions as in previous experiments.

Results

Only correct probe RTs were analyzed (the error rate on probe displays was $28.2 \%$ ). Moreover, RTs had to be longer than $350 \mathrm{~ms}$ and below the Tukey outlier criterion $(1.5$ interquartile ranges above the third quartile, Tukey, 1977; in the present study, this criterion was $4,002 \mathrm{~ms}$, and $4.0 \%$ of the trials were discarded due to the outlier criterion). Excluding outliers and error probes resulted in the elimination of $30.7 \%$ of the trials. The mean RTs and error rates are shown in Table 3. The sequence of the glove conditions showed no main effect or interaction and was dropped for the sake of simplicity.

Reaction times Correct probe RTs were subjected to a 2 (priming condition: IR vs. C) $\times 2$ (gloves: with vs. without gloves) MANOVA. Neither of the main effects was significant, both $F_{\mathrm{s}}<1.81$, both $p \mathrm{~s}>.197$. Yet the interaction between priming and gloves was significant, $F(1,15)=$ $5.68, p=.031, \eta_{\mathrm{p}}^{2}=.28$, indicating different negativepriming effects as a function of whether or not the participant wore gloves. In contrast to the results of Experiment 2, wearing gloves diminished negative priming in the far condition to nonsignificant levels, $M=25 \mathrm{~ms}$ ( $S D 152 \mathrm{~ms}$ ), $t$ $(15)=0.65, p=.526$, whereas when the participants did without the gloves, negative priming of a size comparable to that in the far conditions of the previous experiments was found, $M=-107 \mathrm{~ms}(S D 178 \mathrm{~ms}), t(15)=2.43, p=.028$ (cf. Fig. 2).

Error rates The same MANOVA was conducted on the error data. However, only the main effect of priming

Table 3 Mean reaction times (in milliseconds) and percentages of errors (in parentheses) as a function of priming condition and gloves in Experiment 3

\begin{tabular}{lll}
\hline & \multicolumn{2}{l}{ Gloves } \\
\cline { 2 - 3 } Priming Condition & With & Without \\
\hline Ignored repetition (IR) & $1,743[456]$ & $1,841[382]$ \\
& $(32.2)[22.1]$ & $(28.7)[19.6]$ \\
Control (C) & $1,768[420]$ & $1,734[327]$ \\
& $(26.9)[20.3]$ & $(27.6)[19.6]$ \\
\hline
\end{tabular}

Standard deviations appear in square brackets condition showed a tendency toward significance, $F(1,15)=$ $4.26, p=.057, \eta_{\mathrm{p}}{ }^{2}=.22$, indicating an average negativepriming effect in the error rates, too.

\section{Discussion}

The results of Experiment 3 replicated (for the third time) the tactile negative-priming effect in the far condition without gloves; note that this effect was quite comparable from Experiments 1 to 3 in terms of its size (cf. the bars in Fig. 2). However, when participants wore gloves and had their hands blocked from view in the far condition, negative priming diminished to nonsignificant levels. With respect to the results from the questionnaire, one might have assumed that selection in the far condition would have become easier still whenever the gloves and the cover were used, as the subjectively reported effect of this condition was that the hands seemed to be farther apart and less connected. Thus, tactile negative priming appears to be modulated by the interaction of connectedness and proximity. We will return to this issue in the General Discussion. First, though, we will analyze this interaction by means of an analysis comparing the negative-priming effects from all of the experiments.

Combined analysis of negative-priming effects, experiments $1-3$

We entered the negative-priming effects from all of the experiments as the dependent variable in a multiple regression analysis. Wearing gloves (with vs. without) was entered as a contrast-coded predictor, as well as the distance (near vs. far). In addition, the product term of Distance $\times$ Gloves (accessing the interaction of distance and gloves) was entered as a predictor variable, too. The regression model was significant, $F(3,3)=48.86, p=.005$. Importantly, the product term was also significant, $\beta=.99, p=.002$, reflecting different effects of distance on negative priming as a function of whether or not gloves were worn (cf. Fig. 4).

\section{General discussion}

The results of the present study demonstrate the influence of Gestalt grouping principles on the processing/ignoring of tactile stimuli (specifically, tactile distractors). It has been argued that negative priming with tactile distractors indexes the processing and ignoring of interfering tactile stimuli (Frings et al., 2011; Frings et al., 2008; Frings \& Spence, 2011). In the visual modality, it has been argued that the interference of distractors that can be separated from targets on the basis of Gestalt grouping principles is diminished; this has led to reduced negative-priming effects with visual distractors. In touch, it would appear that the influence of 
Fig. 4 Multiple regression model with the negativepriming effect as the dependent variable and distance between the hands and wearing gloves as independent variables. The product term of both independent variables was significant, reflecting the interaction between distance and whether or not the participant wore gloves

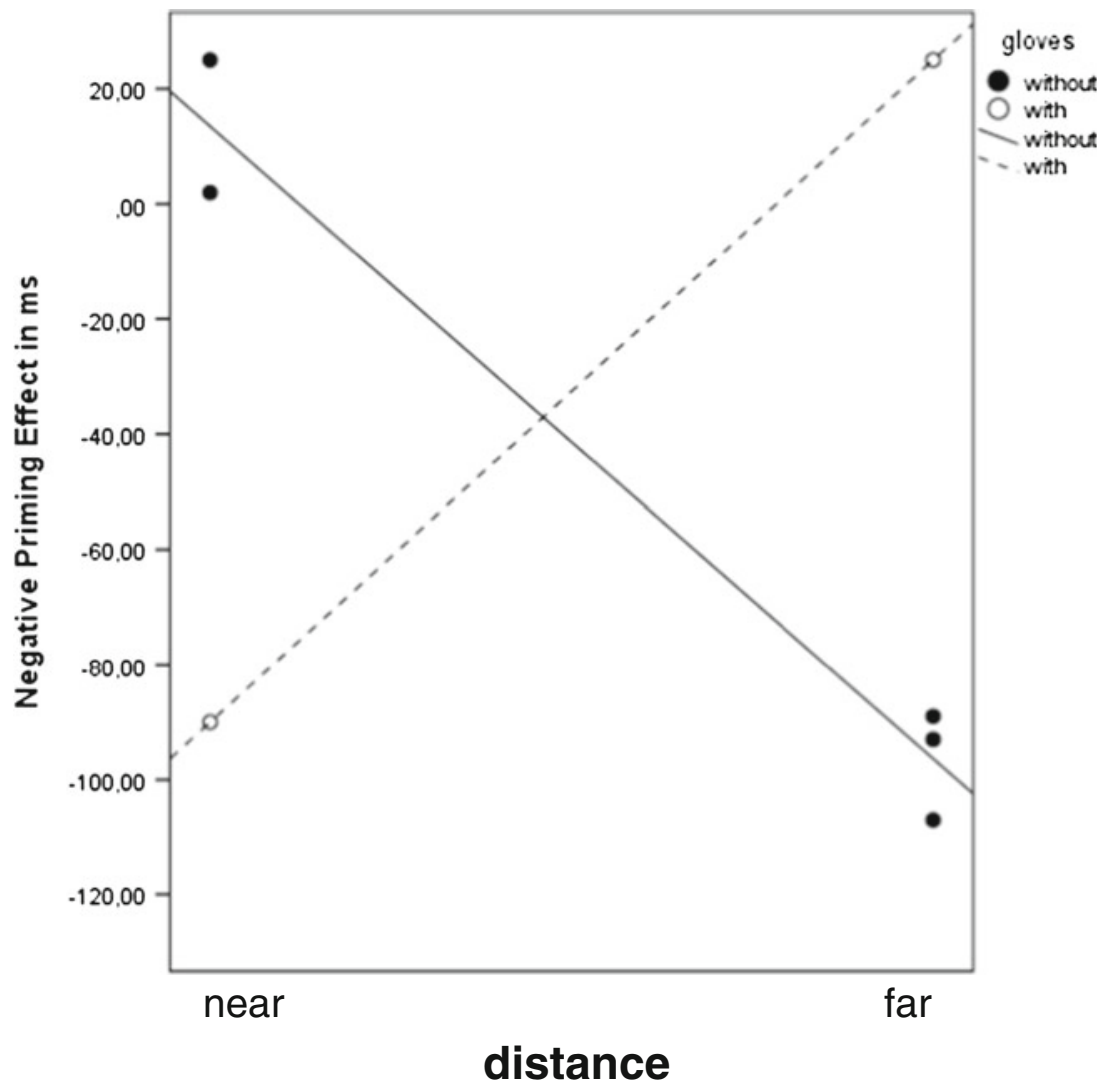

the Gestalt principle of proximity is modulated by other Gestalt principles, such as connectedness. We will discuss our results with respect to these two Gestalt principles.

\section{Connectedness in touch}

Here, using tactile stimuli, we observed, in comparison to vision, a different influence of the Gestalt principle of proximity. Although negative priming diminished to nonsignificant levels when the distance between targets and distractors was small, this effect seemed to be caused by another grouping principle. In fact, if a participant's hands were placed too close together/touching, there may have been a tendency for the participant to group the target and distractor into a single perceptual object. This, in turn, might be expected to prevent, or override, the ignoring of distractors, leading to diminished negative-priming effects in this condition. The effect of touching hands may be understood in terms of the Gestalt principle of connectedness; that is, both stimuli may be encoded as coming from the same object, due to the fact that the hands to which the stimulation was delivered were in contact with each other. This is also what participants reported in the questionnaire. Studies in the visual modality have generated results that are in line with this argument. For example, Behrmann and Tipper (1999) showed that two circles connected by a line (thereby suggesting the impression of a barbell) were grouped into one object, in contrast to two squares that were not connected (despite the spatial distance). In addition, in real life, it seems likely that the simultaneous stimulation of the two hands would often originate from stimulation delivered by the same object (cf. Gallace \& Spence, 2011; O'Regan, 1992). Note, however, that we cannot safely judge whether touching or not touching hands could lead to equivalent differences in the impression that a participant was holding a single object in both hands versus a distinct object in each hand (see, e.g., Gillmeister, Adler, \& Forster, 2010).

Yet, when their hands touched, participants were quite efficient in responding to the tactile targets. Note that the probe RTs may not have provided a valid measure of selection difficulty (even in the control trials), as response hand and target side switches would bias the raw probe RTs. Yet, when looking at the prime RTs (which are free of these biases), in both Experiment 1 and 2 the prime RTs for the condition with touching hands were faster $(2,062 \mathrm{~ms}$ in Exp. 1, and 2,031 ms in Exp. 2) as compared to the conditions with hands not touching (2,137 ms in Exp. 1, and 2,088 vs. 2,074 ms in Exp. 2 for, respectively, the far vs. near-withgloves conditions), all $p \mathrm{~s}<.09$ (one-tailed). Thus, the principle of connectedness leads to efficient selection here; one idea may be that integrating both rhythms into one object may change the selection from object- to feature-based selection, and in turn, the target feature may benefit from the same-object advantage (e.g., Duncan, 1984). This 
reasoning is certainly quite speculative and needs further investigation in the future; nevertheless, it would seem to be an area worth pursuing in future research.

We hasten to add that in the gloves condition - in which we prevented the impression of the hands touching - probably more than just preventing the touching of hands was manipulated. In particular, by using a black cover and conducting the experiment in a dark room, participants also received no visual feedback concerning the position of their hands. Note that Gallace and Spence (2005) had shown previously that the visually perceived distance between the participant's hands can modulate the perception of tactile stimuli delivered to the hands, irrespective of the hands' actual spatial separation. Thus, in the gloves condition, we prevented both the touching of the hands and visual feedback concerning their position, and thereby possibly manipulated, or biased, the participants' perceptions of their hands' locations. Indeed, in a questionnaire on the proprioception of participants' hands, we found that wearing gloves and blocking vision appeared to increase the perceived distance and reduce the connectedness of the participant's hands. We cannot confidently conclude whether one or a combination of these aspects counteracts the Gestalt principle of connectedness in the near condition without gloves. However, note that the intriguing point is that the negativepriming effect diminishes to a nonsignificant level if none of these aspects is controlled for. Therefore, it would seem reasonable to conclude that the distance in external space per se is not what reduces the tactile negative-priming effect, but rather a combination of aspects that we interpret as the Gestalt principle of connectedness, grouping the target and the distractor together into a single perceptual object.

\section{Spatial proximity in touch}

The results reported in the first two experiments of the present study failed to demonstrate any modulation of tactile negative priming as a function of the spatial separation (small vs. large) between the target and distractor hands (at least when the two hands did not directly touch one another). It is important to point out that this null result (of varying spatial separation) might, at least at first glance, appear to contrast with the results of several previous studies that have demonstrated a modulatory effect of varying spatial separation on a variety of tactile tasks (see, e.g., Azañón \& Soto-Faraco, 2008; Gallace \& Spence, 2005; Soto-Faraco, Ronald, \& Spence, 2004). What explains this apparent contradiction?

Well, one plausible possibility here relates to the suggestion that the spatial modulation of tactile performance may simply be more apparent in those studies in which the participants' task is in some sense spatial, as when they are explicitly required to discriminate the spatial location of the target stimulus (e.g., Soto-Faraco et al., 2004). Even the results of tactile temporal order judgment (TOJ) studies can in some sense be thought of as being spatial, in the sense that participants respond by indicating the hand (or location) that was stimulated first (or second; Gallace \& Spence, 2005; Röder et al., 2004). Interestingly, however, as soon as the spatial aspect of the task is removed - as when participants are simply required to judge whether or not the two tactile stimuli (one presented to either hand) were simultaneous, the spatial modulatory effect can dissipate (see Axelrod, Thompson, \& Cohen, 1968). Furthermore, it is also relevant to note here that the results of several spatial attention studies have documented a more pronounced spatial modulation of tactile performance when the participants' task is spatial as compared to when it is nonspatial (see also, e.g., Gallace, SotoFaraco, Dalton, Kreukniet, \& Spence, 2008; Spence \& McGlone, 2001; Spence, Pavani, \& Driver, 2004). The entirely nonspatial nature of the participants' target identification task reported in the present study may therefore have contributed to the apparent absence of a modulatory effect of placing the target and distractor hands near versus far from each other.

Another aspect is that, when modulating the distance between targets and distractors in touch, the distance in external space, as well as the somatosensory distance (i.e., the distance across the skin surface), has to be taken into account. In our experiments, only the distance in external space was varied; thus, we cannot exclude the possibility that the Gestalt principle of proximity may have influenced the processing of tactile distractors when the somatosensory distance between targets and distractors was manipulated. However, recent data from the processing of haptic distractors in a haptic search task has also failed to show an influence of the Gestalt principle of proximity, while the principles of similarity and good continuation were observed in the same study (Overvliet, Krampe, \& Wagemans, 2012). Moreover, it was also argued that the influence of common fate (in the Overliet et al. study, this was the temporal structure of the stimuli) may have overridden the influence of proximity. Generally speaking, it may make sense that, especially for haptics (and maybe for touch stimuli in general), other Gestalt principles would be stronger than proximity in external space, as we can move the effectors with which we experience tactile stimulation in the space around us. For example, if one were exploring quite a big object, it would be maladaptive if, only due to the fact that the hands were far apart, the information from the two hands concerning the object were not to be integrated.

Numerous previous studies have demonstrated that the selection of relevant tactile information - and the ignoring of irrelevant distractor information, no matter whether it is presented in the same or in a different sensory modality-is enhanced (i.e., selection is easier) as the distance between the target and distractors is increased (see Spence \& Gallace, 2007, for a review). Whether the same effect of increasing 
distance would still be obtained if the target and distractor were both presented as part of the same perceptual object is currently not known (though see Driver, 1999, for an analogous debate in the world of visual attention). Here, one might consider the body to be an object much like any other, and to the extent that it is, any object-based attention effects, realized here through the touching of the participants' hands, might be expected to modulate the magnitude of attention effects - as has, in fact, been demonstrated (e.g., Coslett \& Lee, 2004).

Finally, one may explain the effects of wearing gloves and the cover in the far condition of Experiment 3 as an effect of spatial proximity. In fact, when the hands were far apart, negative priming diminished to a nonsignificant level while participants were wearing gloves. As the effects of wearing gloves and blocking vision manipulate the perceived distance of the hands, it can be argued that selection becomes easier still and, in turn, that the magnitude of the negative-priming effect diminishes. A look at the prime RTs indeed shows that in the far condition without gloves, the average RT to correct primes was $1,845 \mathrm{~ms}$, whereas with gloves it was $1,824 \mathrm{~ms}(p<.10)$. Note that probe RTs are not an ideal measure of selection difficulty (even in control trials), as response hand and target side switches bias the raw probe RTs. Thus, as long as the hands do not touch each other, the results of Experiment 3 suggest an influence of spatial proximity in line with that found in vision.

\section{Conclusion}

Taken together, the data reported in the present study suggest that Gestalt principles modulate the ignoring of tactile distractors. The Gestalt principle of proximity hardly seems to play any role at all - at least, in the range of external distances analyzed in the experiments reported here-with respect to the external space in touch. By contrast, the touching of hands modulates whether or not vibrotactile stimuli delivered to both hands are grouped together, thereby influencing the processing and ignoring of distractors.

Author Notes The research reported in this article was supported by a grant of the Deutsche Forschungsgemeinschaft to C.F. and C.S. (FR 2133/5-1). We thank Ben Schneider for his help in conducting the experiment.

\section{References}

Allport, D. A. (1987). Selection-for-action: Some behavioral and neurophysiological considerations of attention and action. In $\mathrm{H}$. Heuer \& A. F. Sanders (Eds.), Perspectives on perception and action (pp. 395-419). Hillsdale, NJ: Erlbaum.

Allport, D. A. (1993). Attention and control: Have we been asking the wrong questions? A critical review of twenty-five years. In D. E.
Meyer \& S. Kornblum (Eds.), Attention and performance XIV (pp. 183-218). Cambridge, MA: MIT Press.

Axelrod, S., Thompson, L. W., \& Cohen, L. D. (1968). Effects of senescence on the temporal resolution of somesthetic stimuli presented to one hand or both. Journal of Gerontology, 23, 191195.

Azañón, E., \& Soto-Faraco, S. (2008). Changing reference frames during the encoding of tactile events. Current Biology, 18, 1044-1049.

Behrmann, M., \& Tipper, S. P. (1999). Attention accesses multiple reference frames: Evidence from visual neglect. Journal of Experimental Psychology. Human Perception and Performance, 25, 83-101.

Bregman, A. S. (1990). Auditory scene analysis: The perceptual organization of sound. Cambridge, MA: MIT Press.

Carter, O., Konkle, T., Wang, Q., Hayward, V., \& Moore, C. (2008). Tactile rivalry demonstrated with an ambiguous apparent-motion quartet. Current Biology, 18, 1050-1054.

Chang, D., Nesbitt, K. V., \& Wilkins, K. (2007). The Gestalt principles of similarity and proximity apply to both the haptic and visual grouping of elements. In W. Piekarski \& B. Plimmer (Eds.), The Eighth Australasian User Interface Conference (AUIC2007), Ballarat, Australia (pp. 79-86). New York, NY: ACM. Conferences in Research and Practice in Information Technology [CRPIT], Vol. 64.

Coslett, H. B., \& Lee, E. (2004). Bare hands and attention: Evidence for a tactile representation of the human body. Neuropsychologia, 42, 1865-1876.

Davis, G., \& Driver, J. (1994). Parallel detection of Kanizsa subjective figures in the human visual system. Nature, 371, 791-793.

Driver, J. (1999). Egocentric and object-based visual neglect. In N. Burgess, K. J. Jeffrey, \& J. O'Keefe (Eds.), The hippocampal and parietal foundations of spatial cognition (pp. 67-89). Oxford, U.K.: Oxford University Press.

Driver, J., \& Baylis, G. C. (1989). Movement and visual attention: The spotlight metaphor breaks down. Journal of Experimental Psychology. Human Perception and Performance, 15, 448-456. doi:10.1037/0096-1523.15.3.448

Duncan, J. (1984). Selective attention and the organization of visual information. Journal of Experimental Psychology. General, 113, 501-517. doi:10.1037/0096-3445.113.4.501

Dyson, B. J. (2009). Perceptual organization. In C. Plack (Ed.), The Oxford handbook of auditory science: Auditory perception (pp. 177-206). Oxford, U.K.: Oxford University Press.

Egly, R., Driver, J., \& Rafal, R. D. (1994). Shifting visual attention between objects and locations: Evidence from normal and parietal lesion subjects. Journal of Experimental Psychology. General, 123, 161-177. doi:10.1037/0096-3445.123.2.161

Fox, E. (1995). Negative priming from ignored distractors in visual selection: A review. Psychonomic Bulletin \& Review, 2, 145-173.

Fox, E. (1998). Perceptual grouping and visual selective attention. Perception \& Psychophysics, 60, 1004-1021.

Frings, C., Amendt, A., \& Spence, C. (2011). When seeing doesn't matter: Assessing the after-effects of tactile distractor processing in the blind and the sighted. Journal of Experimental Psychology. Human Perception and Performance, 37, 1174-1181.

Frings, C., Bader, R., \& Spence, C. (2008). Selection in touch: Negative priming with tactile stimuli. Perception \& Psychophysics, 70, 516-523.

Frings, C., \& Rothermund, K. (2011). To be or not to be. .. included in an event file: Integration and retrieval of distractors in stimulusresponse episodes is influenced by perceptual grouping. Journal of Experimental Psychology: Learning, Memory, and Cognition, 37, 1209-1227.

Frings, C., \& Spence, C. (2010). Crossmodal congruency effects based on stimulus identity. Brain Research, 1354, 113-122. 
Frings, C., \& Spence, C. (2011). Increased perceptual and conceptual processing difficulty makes the immeasurable measurable: Negative priming in the absence of probe distractors. Journal of Experimental Psychology. Human Perception and Performance, 37, $72-84$.

Frings, C., \& Wühr, P. (2007). Prime-display offset modulates negative priming only for easy-selection tasks. Memory \& Cognition, 35, 504-513.

Fuentes, L. J., Humphreys, G. W., Agis, I. F., Carmona, E., \& Catena, A. (1998). Object-based perceptual grouping affects negative priming. Journal of Experimental Psychology. Human Perception and Performance, 24, 664-672.

Gallace, A., Soto-Faraco, S., Dalton, P., Kreukniet, B., \& Spence, C. (2008). Response requirements modulate tactile spatial congruency effects. Experimental Brain Research, 191, 171-186. doi:10.1007/s00221-008-1510-x

Gallace, A., \& Spence, C. (2005). Visual capture of apparent limb position influences tactile temporal order judgments. Neuroscience Letters, 379, 63-68.

Gallace, A., \& Spence, C. (2011). To what extent do Gestalt grouping principles influence tactile perception? Psychological Bulletin, $137,538-561$.

Gallace, A., \& Spence, C. (in press). In touch with the future. Oxford, U.K.: Oxford University Press.

Gillmeister, H., Adler, J., \& Forster, B. (2010). Object-guided spatial attention in touch: Holding the same object with both hands delays attentional selection. Journal of Cognitive Neuroscience, 22, 931-942.

Haggard, P., Kitadono, K., Press, C., \& Taylor-Clarke, M. (2006). The brain's fingers and hands. Experimental Brain Research, 172, 94 102

Harrar, V., \& Harris, L. R. (2007). Multimodal Ternus: Visual, tactile, and visuo-tactile grouping in apparent motion. Perception, 36, $1455-1464$.

Houghton, G., \& Tipper, S. P. (1994). A model of inhibitory mechanisms in selective attention. In D. Dagenbach \& T. H. Carr (Eds.), Inhibitory processes in attention, memory and language (pp. 53112). San Diego, CA: Academic Press.

Houghton, G., \& Tipper, S. P. (1996). Inhibitory mechanisms of neural and cognitive control: Applications to selective attention and sequential action. Brain and Cognition, 30, 2043.

Johnston, W. A., \& Dark, V. J. (1986). Selective attention. Annual Review of Psychology, 37, 43-75.

Julesz, B. (1971). Foundations of cyclopean perception. Chicago, IL: Chicago University Press.

Kahneman, D., \& Henik, A. (1981). Perceptual organization and attention. In M. Kubovy \& J. R. Pomerantz (Eds.), Perceptual organization (pp. 181-211). Hillsdale, NJ: Erlbaum.

Kahneman, D., \& Treisman, A. (1984). Changing views of attention and automaticity. In R. Parasuraman \& D. R. Davies (Eds.), Varieties of attention (pp. 26-61). San Diego, CA: Academic Press.

Koffka, K. (1935). Principles of Gestalt psychology. New York, NY: Harcourt, Brace, \& World.

Köhler, W. (1930). Gestalt psychology. London, U.K.: G. Bell \& Sons.

Lakatos, S., \& Shepard, R. N. (1997). Constraints common to apparent motion in visual, tactile, and auditory space. Journal of Experimental Psychology. Human Perception and Performance, 23, $1050-1060$.

Lamers, M. J. M., \& Roelofs, A. (2007). Role of Gestalt grouping in selective attention: Evidence from the Stroop task. Perception \& Psychophysics, 69, 1305-1314. doi:10.3758/BF03192947

Moeller, B., \& Frings, C. (2011). Remember the touch: Tactile distractors retrieve previous responses to targets. Experimental Brain Research, 214, 121-130.
Neill, W. T. (1997). Episodic retrieval in negative priming and repetition priming. Journal of Experimental Psychology: Learning, Memory, and Cognition, 23, 1291-1305. doi:10.1037/02787393.23.6.1291

O’Regan, J. K. (1992). Solving the "real" mysteries of visual perception: The world as an outside memory. Canadian Journal of Psychology, 46, 461-488. doi:10.1037/h0084327

Overvliet, K. E., Krampe, R. T., \& Wagemans, J. (2012). Perceptual grouping in haptic search: The influence of proximity, similarity, and good continuation. Journal of Experimental Psychology. Human Perception and Performance, 38, 817-821.

Pashler, H. E. (1998). The psychology of attention. Cambridge, MA: MIT Press.

Reynolds, M., Kwan, D., \& Smilek, D. (2010). To group or not to group: An ecological consideration of the Stroop effect. Experimental Psychology, 57, 275-291.

Röder, B., Rösler, F., \& Spence, C. (2004). Early vision impairs tactile perception in the blind. Current Biology, 14, 121-124.

Smith, B. (Ed.). (1988). Foundations of Gestalt theory. Munich, Germany: Philosophia Verlag.

Soto-Faraco, S., Ronald, A., \& Spence, C. (2004). Tactile selective attention and body posture: Assessing the multisensory contributions of vision and proprioception. Perception \& Psychophysics, 66, 1077-1094. doi:10.3758/BF03196837

Spence, C. (2010). Crossmodal attention. Scholarpedia, 5, 6309. doi: $10.4249 /$ scholarpedia.6309

Spence, C., \& Gallace, A. (2007). Recent developments in the study of tactile attention. Canadian Journal of Experimental Psychology, 61, 196-207.

Spence, C., \& McGlone, F. P. (2001). Reflexive spatial orienting of tactile attention. Experimental Brain Research, 141, 324-330.

Spence, C., Pavani, F., \& Driver, J. (2004). Spatial constraints on visual-tactile cross-modal distractor congruency effects. Cognitive, Affective, \& Behavioral Neuroscience, 4, 148-169. doi:10.3758/ CABN.4.2.148

Tipper, S. P. (1985). The negative priming effect: Inhibitory priming by ignored objects. Quarterly Journal of Experimental Psychology, $37 A, 571-590$.

Tipper, S. P. (2001). Does negative priming reflect inhibitory mechanisms? A review and integration of conflicting views. Quarterly Journal of Experimental Psychology, 54A, 321-343.

Tukey, J. W. (1977). Exploratory data analysis. Reading, MA: Addison-Wesley.

von Ehrenfels, C. F. (1890). Ueber Gestaltqualitaeten [On the qualities of form]. Vierteljahresschrift für wissenschaftliche Philosophie, 14, 249-292.

Wagemans, J. (Ed.). (2012). The Oxford handbook of perceptual organization. Oxford, U.K.: Oxford University Press.

Wagemans, J., Elder, J. H., Kubovy, M., Palmer, S. E., Peterson, M. A., Singh, M., \& von der Heydt, R. (2012). A century of Gestalt psychology in visual perception: I. Perceptual grouping and figure-ground organization. Psychological Bulletin, $138,1218-1252$.

Wertheimer, M. (1912). Experimentelle Studien ueber das Sehen von Bewegung [Experimental studies on the visual perception of movement]. Zeitschrift für Psychologie, 61, 161-265.

Wertheimer, M. (1938). Laws of organization in perceptual forms. In W. D. Ellis (Ed.), A source book of Gestalt psychology (pp. 7188). London, U.K: Routledge \& Kegan Paul. Original work published 1923.

Yeh, Y.-Y., \& Chao, H.-F. (2004). Probe distractors can influence negative priming by perceptual grouping. Perception \& Psychophysics, 66, 208-218.

Zmigrod, S., Spapé, M., \& Hommel, B. (2009). Intermodal event files: Integrating features across vision, audition, taction, and action. Psychological Research, 73, 674-684. 